\title{
Substrate Integrated Circular Cavity Resonator Filled with Nano-Fibrillated Cellulose for Humidity Detection
}

\author{
Majid Ndoye ${ }^{1}$, Benoit Bideau ${ }^{1}$, Aina Heritiana Rasolomboahanginjatovo ${ }^{1}$, Éric Loranger ${ }^{1}$, \\ Dominic Deslandes ${ }^{2}$, Frédéric Domingue ${ }^{1}$
}

\author{
${ }^{1}$ Université du Québec à Trois-Rivières (UQTR), Trois-Rivières (QC), Canada \\ ${ }^{2}$ École de technologie supérieure (ETS), Montréal (QC), Canada \\ *Corresponding author: E-mail: majid.ndoye@uqtr.ca
}

Received: 11 August 2017, Revised: 13 December 2018 and Accepted: 14 December 2018

DOI: 10.5185/amlett.2019.1927

www.vbripress.com/aml

\begin{abstract}
In this work, a novel microwave sensor fully based on Substrate Integrated Waveguide (SIW) technology filled with nanofibrillated cellulose for humidity detection is presented for the very first time. The proposed structure consists of a circular SIW cavity resonator perturbed by the inclusion of nano-fibrillated cellulose inside the cavity. Due to the presence of humidity, the relative permittivity of the eco-friendly dielectric, which is known as a humidity sensitive material, changes, leading to a shift of the resonance frequency of the Substrate Integrate Cavity Circular Resonator (SICCR). The proposed humidity sensor structure operates between 4.28 to $4.32 \mathrm{GHz}$ and exhibits a frequency shift of around $20 \mathrm{MHz}$ for relative humidity in the range of $11.7 \%$ to $91 \%$ RH. The proposed sensing device operates with very low-cost sustainable and renewable material, is simple to manufacture, co-integrates with existing microwave planar circuits and has the advantage of demonstrating high sensitivity performance. Copyright $($ ) VBRI Press.
\end{abstract}

Keywords: Nano-fibrillated cellulose, humidity sensor, microwave cavity resonator, substrate integrated waveguides (SIW).

\section{Introduction}

In recent years, terms like "going green" and "ecofriendly" have become one of the hot topics in major influential conferences and international summits. With the current warning on the degradation of the environment, on global warming and pollution, the trend both in industrial and research field is to be more selective with materials and products used by prioritizing those that are ecological and biodegradable.

As most of the leading scientific domains such as Materials Science and Engineering, Bioengineering, Civil and Mining Engineering, Biochemistry and Chemical Engineering, the Electrical and Industrial Engineering field has experienced the advent of biodegradable materials into end product devices, notably into humidity sensors.

Humidity sensing has gained an increasing interest in health and well-being related applications aiming to provide and ensure clean environment. Humidity sensors are widely used not only for indoor air quality monitoring for households and medical institutes, but also in moisture monitoring for food storage and industrial applications involving moisture control such as chemical gas purification, film desiccation, paper and textile manufacturing.
To comply with the requirements of the current in demand humidity sensing performance requirements, most of the humidity sensors on the market are active $[1,2]$ and integrate within their structures materials which are sensitive to humidity such as dielectric $[3,4]$, inorganic polymers [5, 6], alumina thin films [7], etc. However, due to the complex challenge of integrating power supply, analog to digital converters (ADC) and amplifiers, a great deal of effort has been made to develop passive sensors. In addition to their fossil attributes which may contaminate the structure when decomposing, most of the humidity sensitive materials are difficult to synthesize. They are made and integrated to the structure through complex fabrication process involving additional contaminating hazardous chemical materials. Therefore, despite the availability of several types of sensors on the market, it is still essential to find more technological solutions that allow humidity sensing devices to satisfy a wide range of requirements.

The humidity sensor proposed in this work is highly sensitive and uses "eco-friendly" sensitive materials. The proposed device is fully passive and is expected to be fabricated and commercialized at low cost.

Carbohydrate made of a linear chain of D-glucose molecules, cellulose $\left(\mathrm{C}_{6} \mathrm{H}_{10} \mathrm{O}_{5}\right) \mathrm{n}$ is the most abundant material on earth. Cellulose fibers are mainly extracted 
from wood and are $100 \%$ natural organic polymer. Cellulose is environmentally friendly, inexpensive, renewable, biodegradable and biocompatible. Using cellulose-based materials is advantageous since cellulose shows high water and humidity sensitivity. Indeed, cellulose films have very poor barrier properties to water vapor thanks to its hydrophilicity. Many studies investigated the effect of the humidity on microcrystalline cellulose [8] and the influence of temperature and humidity on cellulose nanocrystals [9].

Cellulose acetate butyrate (CAB) and carboxymethyl cellulose were tested through exposure to humidity. The results revealed their high capability for humidity detection.

Few smart semi-conductor humidity sensors using cellulose derivatives, namely cellulose acetate-copper oxide [10] and cellulose nanocrystal-graphene oxide [11], as humidity sensitive materials have been fabricated and investigated. However, most of the cellulose derivatives based sensitive materials which were tested are derivatives of inorganic materials and are not fully biodegradable. Indeed, $\mathrm{CAB}$ and the other fabricated sensors were made through complex manufacturing process handling active technology and making their cost high.

The Substrate Integrated Waveguide (SIW) technology has been widely demonstrated in many research articles for telecommunications components and systems [12]. The popularity of SIW technology in the field of millimeter and passive systems comes from its advantage of being a low-cost technology, its highquality factor, low insertion loss and the easy cointegration with other planar circuits. Recently published articles have also demonstrated that SIW can be used for sensing applications. For example, SIW resonators have been used to detect or characterize a fluid in a system or to characterize materials [13]. To the best knowledge of the authors, no investigation of fully biodegradable materials for humidity sensing in passive microwave devices has been reported.

This work proposes a Substrate Integrated Circular Cavity Resonator (SICCR) filled with nano-fibrillated cellulose (TOCN) for humidity detection. Our research focuses specifically on a general study of the design and realization of the SICCR-based humidity sensor. Experimental humidity validations have been performed on the circular resonator sensor sample in the range of $11.7 \%$ to $85 \% \mathrm{RH}$. The proposed sensing device operates using a very low-cost sustainable and renewable material that is easy to manufacture, co-integrates well with existing microwave planar circuits and has the advantage of demonstrating high sensitivity performance with excellent sensing properties at a very low cost.

\section{Sensor structure and sensing concept}

\section{Sensor structure}

Fig. 1 shows the structure of the substrate integrated cavity circular resonator of the proposed humidity sensor.

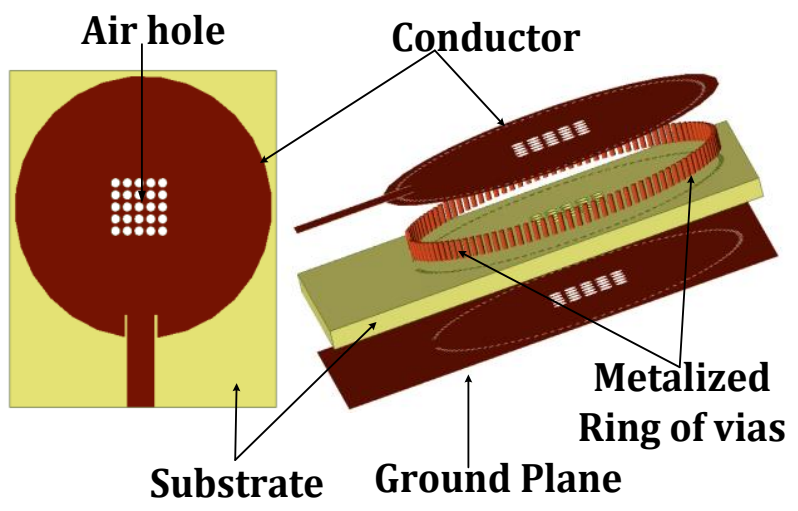

Fig. 1. Structure of the SIW-based Cavity Circular Resonator of the proposed humidity sensor.

The metalized ring of vias placed inside the dielectric substrate and plated with copper on both side of the substrate forms the electric side-wall of the circular cavity resonator. The SICCR is considered as a short-circuited waveguide. The eigen-mode exploited in the cylindrical cavity is either the transverse electric (TE) mode or the transverse magnetic (TM) mode.

The TE mode corresponds to the propagation of an electromagnetic wave whose electric field is transverse and thus contained in the right sectional plane of the guiding structure while the magnetic field is propagating in the propagation direction of the guide. $E_{z}=0$ and $H_{z} \neq 0$ for the TE mode. Vice versa, $E_{z} \neq 0$ and $H_{z}=0$ for the TM mode. As mentioned in [14], only the TM mode is excited in this case study. If:

- $(2 a / d)<0.985$, therefore, the dominant mode of the proposed structure is TE111,

- $(2 a / d)>0.985$, the dominant mode is TM010.

The TM010 (Fig. 3) mode is widely used for the design of TM single mode resonators. In both cases, the resonance frequency of the circular cavity resonator corresponds to the lowest resonance frequency.

\section{Operating principle}

The operating principle of the humidity sensor is based on the humidity sensitivity properties of the nanofibrillated celluloses (TOCN) (Fig. 2) introduced inside of the SICCR.

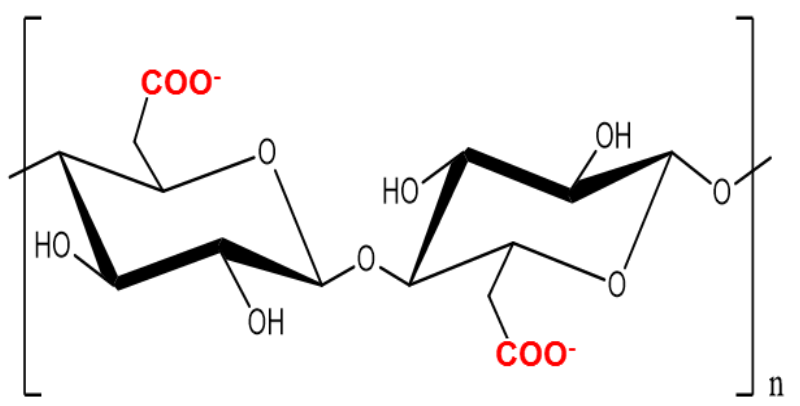

Fig. 2. Structure of Nano-Fibrillated Cellulose Oxidized by TEMPO reaction. 
The nano-fibrillated cellulose used in this study was obtained by TEMPO oxidation reaction of wood kraft pulp, a Regio-selective reaction that chemically modifies the primary alcohol groups of cellulose [15-16]. The advantages of using oxidized cellulose fibers include their large specific surface area, good adherence thanks to the surface charges and their high-water sensitivity [17]. Indeed, TOCN films are vulnerable to water. The nano-fibrillated celluloses display a typical high water affinity associated with a fast decrease in the contact angle, due to their high hydrophilic character which exerts high attraction on free water molecules through the establishment of hydrogen bonds (Fig. 3) [18].

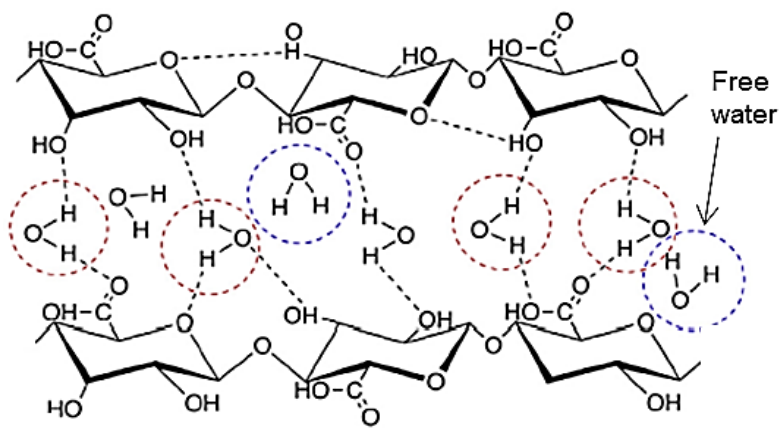

Fig. 3. Interactions of Nano-Fibrillated Cellulose with free water.

Thus, due to the presence of humidity, the relative permittivity of the eco-friendly dielectric changes, leading to a shift of the resonance frequency of the SIW resonator. The detection principle of the SICCR humidity sensor is based on this frequency shift which later on is used to estimate the humidity percentage.

\section{Theory and electromagnetic analysis}

\section{Resonance frequency}

The resonance frequency $f_{n m l}$ of the $\mathrm{TM}_{\mathrm{nml}}$ mode of a SIW cylindrical resonator is [19]:

$$
f_{n m l}=\frac{c}{2 \pi \sqrt{\varepsilon_{r}}} \sqrt{\left(\frac{\rho_{n m}}{a}\right)^{2}+\left(\frac{l \pi}{d}\right)^{2}}
$$

where, $\varepsilon_{r}$ is the relative permittivity of the substrate, $c$ is the speed of light, $a$ and $d$ are respectively the inner radius and the height of the resonant cavity, $\rho_{n m}$ is the $\mathrm{m}$-th root of the first kind Bessel function $J_{n}(x)$.The indices $n, m$ and $l$ are the numbers of half-wavelength variations in the standing-wave pattern of the cylindrical $\mathrm{TM}_{\mathrm{nml}}$ mode. Functionalizing the SIW resonant cavity with a sensitive dielectric implies introducing a small dielectric sample into the original substrate. The introduction of a foreign material in the cavity of the resonator leads to a shift of the resonance frequency. The effect of such perturbations on the cavity resonator can be estimated. An approximated value of the perturbed resonance frequency can be calculated using the perturbation method (2) [19].

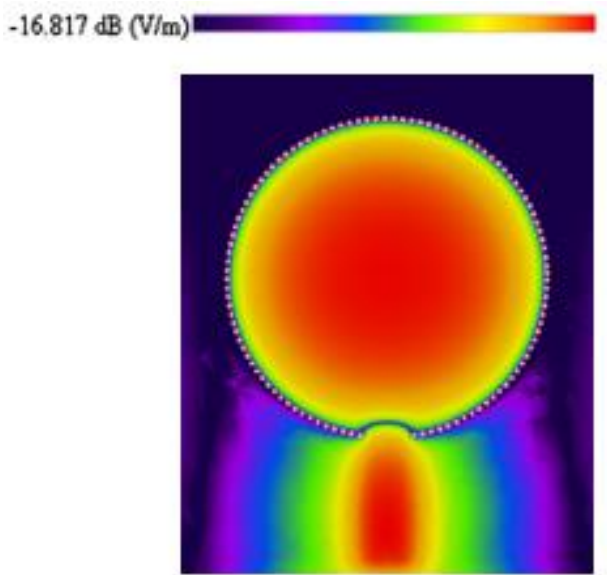

Fig. 4. Magnitude of the electric field distribution in a circular SIW for mode TE101

As shown in Fig. 4, the electric field distribution for the dominant resonance mode $\mathrm{TM}_{010}$ into the circular resonator is more intense in the middle of the structure.

The best sensitivity of the SIW resonator is when the functionalized region is set in the maximum E-field area of the different explored modes [20].

For TM modes, the unperturbed electric field $E_{z}$ is given by (2) [21]:

$E_{z}=\sqrt{\frac{\mu}{\varepsilon}} \frac{\rho_{n m}}{a}\left(\left(\frac{\rho_{n m}}{a}\right)^{2}+\left(\frac{n \pi}{d}\right)^{2}\right)^{-\frac{1}{2}} J_{n}\left(\frac{\rho_{n m}}{a} \rho\right) \cos n \theta \cos \frac{l \pi z}{d}$

For a dielectric perturbation $\Delta \varepsilon$, the variation of the resonance frequency $f_{r}$ is described in equation (3). At the resonance, we have $\varepsilon|E|^{2}=\mu|H|^{2}$. In absence of variation of the relative permeability, $\mu=1$ and $\Delta \mu=0$.

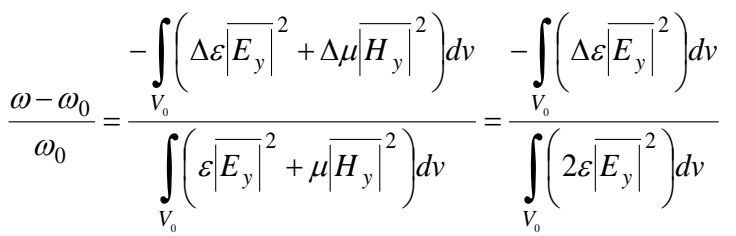

while $E_{0}=\sqrt{\frac{\mu}{\varepsilon}} \frac{\rho_{n m}}{a}\left(\left(\frac{\rho_{n m}}{a}\right)^{2}+\left(\frac{l \pi}{d}\right)^{2}\right)^{-1 / 2}$.

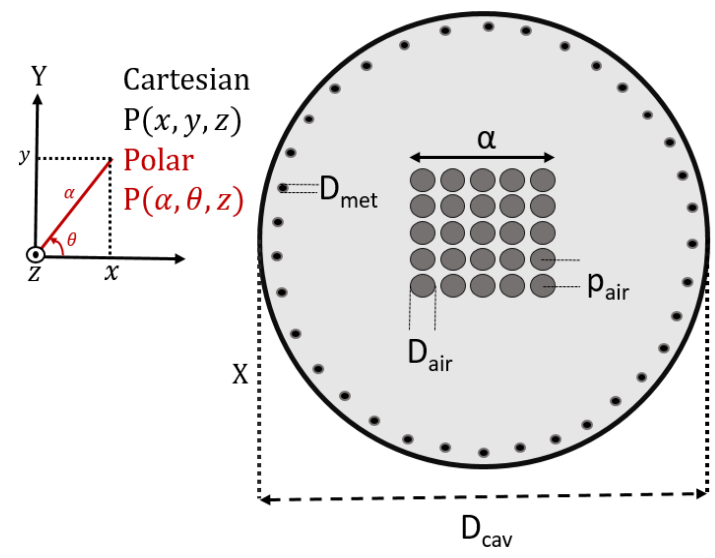

Fig. 5. Dimensions of a SIW structure. 
The theoretical expression of the resonance frequency for the $\mathrm{TM}_{010}$ mode is a function of the SIW structure's dimensions illustrated in Fig. 5. The resonance frequency of the cavity resonator is calculated by using the dielectric perturbation technique.

$$
\begin{aligned}
f=\frac{-\Delta \varepsilon \alpha^{2} f_{0}}{4 \varepsilon \alpha^{2}\left(J_{n}^{\prime}\left(\rho_{n m}\right)\right)^{2}} & \left(\left(J_{n}^{\prime}\left(\frac{\rho_{n m}}{2 a} \alpha\right)\right)^{2}\right. \\
+ & \left.\left(1-\frac{4 a^{2} n^{2}}{\rho_{n m}^{2} \alpha^{2}}\right)\left(J_{n}\left(\frac{\rho_{n m}}{2 a} \alpha\right)\right)^{2}\right) \\
+ & f_{0}
\end{aligned}
$$

where, $f_{0}$ is the unperturbed frequency, $\Delta \varepsilon=\varepsilon_{\text {rmat }}-$ $\varepsilon_{\text {rsub }}, \varepsilon_{\text {rmat }}$ is the effective permittivity and $\varepsilon_{r s u b}$ is the permittivity of the substrate. The value of $\varepsilon_{\text {rmat }}$ is determined by using the Bruggeman's effective medium theory (EMT) [22].

Equation (4) represents the general expression of the resonance frequency of the SICCR when the sensitive region is set at the center of the cavity of the resonator. As illustrated in Fig. 3, this configuration is ideal for the $\mathrm{TM}_{101}$ electric field distribution which is maximum in the center of the cavity.

Table 1. Design Parameter Values of The Sensor (Millimeters).

\begin{tabular}{c|c|c|c|c|c}
\hline Parameters & Dcav & $\mathrm{W}_{0}$ & $\mathrm{~L}_{0}$ & $\mathrm{D}_{\text {met }}$ & $\mathrm{D}_{\text {air }}$ \\
\hline Values & 28 & 3.5 & 9 & 0.5 & 1.2 \\
\hline
\end{tabular}

\section{Device and measurement}

The dimensions of the proposed humidity detection structure are detailed in Table 1. Fig. 6 shows the fabricated structure using the dielectric substrate ROGER4003C $\quad\left(\varepsilon_{r}=3.55, \quad \tan \delta=0.0027, \quad h=\right.$ $1.524 \mathrm{~mm})$. The inner radius of the cavity of the resonator prototypes is $14 \mathrm{~mm}$. The humidity detector sensor was made using a standard PCB manufacturing technique. The diameter $d$ of the metalized posts is $0.5 \mathrm{~mm}$, while the pitch of the posts is $1 \mathrm{~mm}$. The air holes were drilled with a diameter $D_{\text {air }}=1.2 \mathrm{~mm}$ and a pitch $p_{\text {air }}=1.5 \mathrm{~mm}$.

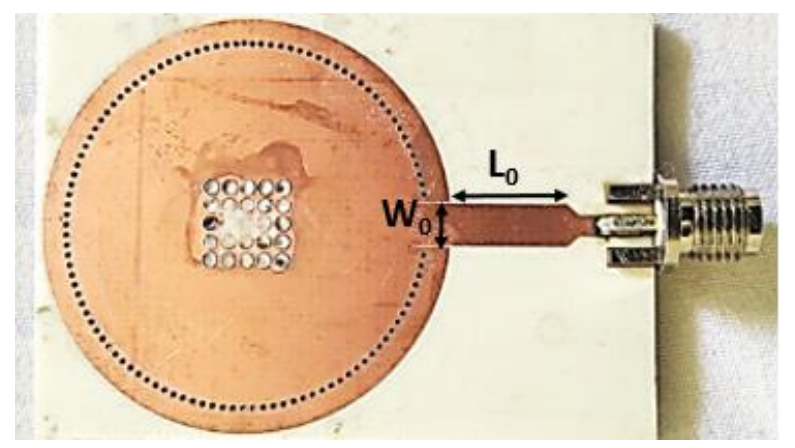

Fig. 6. Fabricated SICCR-based humidity sensor.

Using equation (4) as well as the parameters listed in Table 1, the theoretical resonance frequency is found to be $f_{r}=4.381 \mathrm{GHz}$. A comparison between the simulated and measured S11-parameter of the SICCR sensor filled with air holes is shown in Fig. 7.

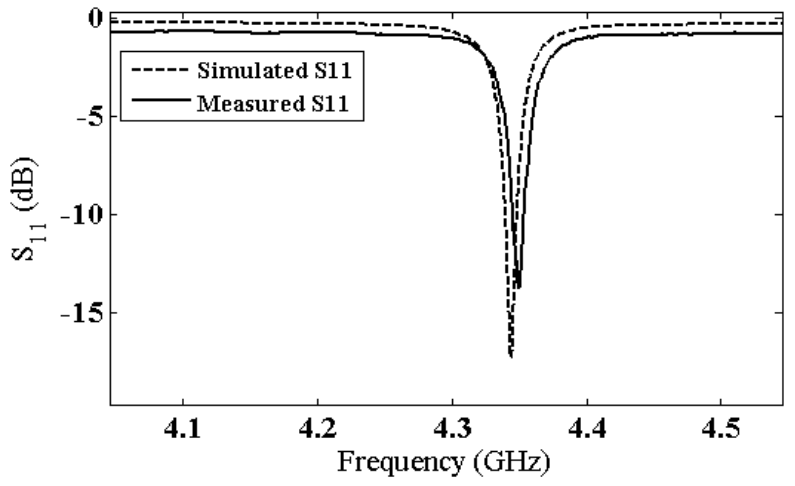

Fig. 7. Simulated and Measured S11-Parameters of SICCR.

The simulated values and the experimental results match very well. The measured operating frequency of the cavity resonator for the fundamental mode $\mathrm{TM}_{101}$ is $4.353 \mathrm{GHz}$, while the simulation predicts $4.344 \mathrm{GHz}$.

The measured quality factor $(\mathrm{Q})$ of the sensor device is 294.23. The measurements were taken at ambient humidity $\sim 24 \% \mathrm{RH}$.

\section{Experimental validation}

\section{Set up and test bench}

To demonstrate the humidity sensing capability of the proposed SICCR-based sensor, the device was placed in a controlled humidity environment test bench. The measurement set up has been configured to expose the humidity sensor to RH environments using saturated salt solution as humidity source. The sodium chloride - based salt solution was put on a plate and placed inside a hermetic box. This testing method has been used and approved for humidity tests in several articles [23, 24], and allows the operator to control and maintain the relative humidity in the air-tight test box at the desired level.

\section{Experimental results}

The magnitude of the S11 parameter of the proposed sensor has been measured with the VNA to determine how much the relative humidity in the test box affects the resonance frequency. The response of the sensor while exposed to humidity concentration ranging from 11.7 RH\% to 91 RH\% is shown in Fig. 8.

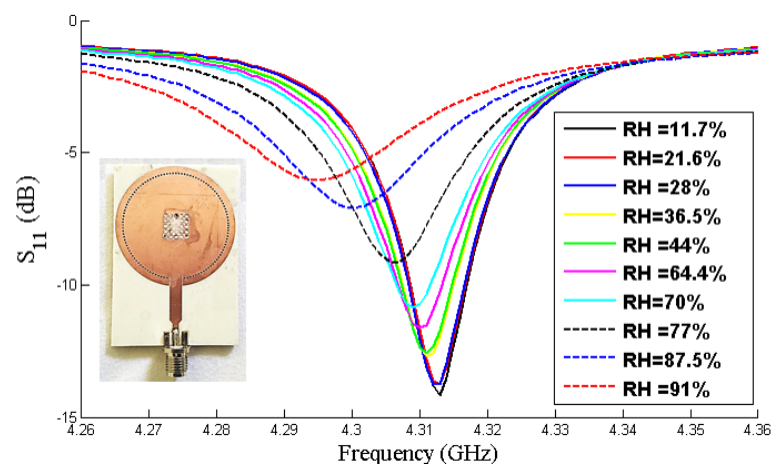

Fig. 8. Shift of frequency resonance of the SICCR depending on relative humidity $\% \mathrm{RH}$. 
For each measurement, the sensor device was connected to the VNA and kept inside the box where the saturated salt solution was placed until the relativity humidity level stops fluctuating. The full stabilization of the system takes approximately 10 minutes. The device demonstrates an excellent repeatability and stability. The response time and the recovery time of the sensor are relatively fast, respectively less than $2 \mathrm{~s}$ and about $4 \mathrm{~s}$.

The presence of humidity leads to a shift of the resonance frequency of the device. A maximum frequency shift of $19.6 \mathrm{MHz}$ is obtained when the air inside the box is $91 \% \mathrm{RH}$. The sensitivity $S[\mathbf{2 5}]$ has been found to be $\mathrm{S}=273.5 \mathrm{kHz} / \mathrm{RH} \%$ for humidity concentration ranging from $11.7 \mathrm{RH} \%$ to $91 \mathrm{RH} \%$. The proposed SCCR-based humidity detector filled with TOCN stands out from other humidity sensors for its high sensitivity, high accuracy and environmentally friendly properties. Thanks to its hydrophilic character from the presence of polar groups on surface, the TOCN adsorbs the water molecules in the surrounding air up to equilibrium with the outside. The equilibrium varies depending on humidity rate.

As a resonator, the proposed humidity sensor not only has a high Q factor and a low insertion loss, but is also low cost and easy to integrate with planar circuits.

\section{Conclusion}

This work presents the very first microwave SICCR filled with eco-friendly Nano-Fibrillated Cellulose for humidity detection. The theoretical model of the perturbed resonance frequency of the circular SIW cavity resonator was estimated using the perturbation method. The sensor exhibits a sensitivity of $273.5 \mathrm{kHz} / \mathrm{RH} \%$ while tested in humidity conditions ranging from $11.7 \% \mathrm{RH}$ to $91 \% \mathrm{RH}$. The environmentally friendly SCCR-based humidity detector is original for its high Q factor, low insertion loss, high sensitivity, low cost and its co-integrability with planar circuits.

\section{References}

1. Abdolrazzaghi, M.; Daneshmand M.; "Enhanced Q double resonant active sensor for humidity and moisture effect elimination, 2016 IEEE MTT-S International Microwave Symposium (IMS), San Francisco, CA, 2016, 1.

2. Islam, T.; Gangopadhyay, A.; Zaheeruddin, An oscillator based active bridge circuit for converting capacitance change into frequency for capacitive humidity sensor, International Conference on Communications, Devices and Intelligent Systems (CODIS), Kolkata, 2012, 480.

3. Gonçalves, R.; Pinho, P.; Carvalho, N. B.; Tentzeris, M. M., Humidity passive sensors based on UHF RFID using cork dielectric slabs, 9th European Conference on Antennas and Propagation (EuCAP), Lisbon, 2015, 1.

4. Raza, E.; Asif, M.; Aziz, F.; Azmer, M. I.; Malik, H. A.; Teh, C. H.; Najeeb, M. A.; Zafar, Q.; Ahmad, Z.; Wahab, F.; Daik, R.; Sarih, N. M.; Supangat, A.; Sulaiman, K., Sens. Actuators, B, 2016, 235, 146.

5. Jiang, K.; Zhao, H.; Fei, T.; Dou, H.; Zhang, T., Sens. Actuators, $B$, 2016, 222, 440 .

6. Yuan, L.; Hui, H.; Lingling, W.; Daoping, C.; Bin, L.; Dandan, W.; Qiuhong, L.; Taihong, W., Sens. Actuators, B, 2016, 223, 730 .

7. Sharma, K.; Islam, S. S., Sens. Actuators, B, 2016, 237, 443.
8. Hammes, M. V.; Englert, A. H.; Zapata Noreña, C. P.; Medeiros Cardozo, N. S., Colloids Surf., A, 2016, 500, 118.

9. Wu, Q.; Meng, Y.; Concha, K.; Wang, S.; Li, Y.; Ma, L.; Fu, S., Sens. Actuators, B, 2013, 48, 28.

10. Chani, M. T. S.; Karimov, K. S.; Khan, S. B.; Asiri, A. M., Sens. Actuators, A, 2016, 246, 58

11. Kafy, A.; Md. I. R. Shishir, A. A.; Kim, H. C.; Yun, Y.; Kim, J., Sens. Actuators, A, 2016, 247, 221.

12. Deslandes, D., Design equations for tapered microstrip-toSubstrate Integrated Waveguide transitions, IEEE MTT-S International Microwave Symposium Digest, 23, 2010.

13. Sama, N. Y.; Ndoye, M.; Deslandes, D.; Domingue, F., Partiallyopen SIW resonator for microwave characterization of particulate dielectrics: Effect of interaction with gases," IEEE International Workshop on Electromagnetics: Applications and Student Innovation Competition (iWEM), Nanjing, 2016.

14. Karmel, P. R.; Colef, G. D.; Camisa, R. L., Introduction to Electromagnetic and Microwave Engineering, Wiley, UK, 1998.

15. Paquin, M.; Loranger, E.; Hannaux, V.; Chabot, B.; Daneault, C., Ultrason. Sonochem., 2013, 20, 103.

16. Rattaz, A.; Mishra, S. R.; Chabot, B.; Daneault, C., Cellulose, 2011, 18,585 .

17. Syverud, K.; Stenius, P., Cellulose, 2009, 16, 75.

18. Bideau, B.; Bras, J.; Saini, S.; Daneault, C.; Loranger E., Mater Sci. Eng., C, 2016, 69, 977.

19. Pozar, D. M., Microwave Resonators, Pozar, D. M. (Ed.); Wiley: USA, 2012, pp 272-306.

20. Ndoye, M.; El Matbouly, H.; Sama, Y.; Deslandes, D.; Domingue, F., Sens. Actuators, B, 2016, 251, 198.

21. Kinzer, J. P.; Wilson, I. G., The Bell System Technical Journal, 1947, 26, 410.

22. Karkkainen, K. K.; Sihvola, A. H.; Nikoskinen, K. I., IEEE Trans. Geosci. Electron., 2000, 38, 1303.

23. Zhuang, Z.; Qi, D.; Zhao, C.; Na, H., Sens. Actuators, B, 2016 , 236,701 .

24. Su, Y.; Xie, G.; Wang, S.; Tai, H.; Zhang, Q.; Du, H.; Zhang, H.; Du, X.; Jiang, Y., Sens. Actuators, B, 2017, 251, 144.

25. Chang, K.; Kim, Y. H.; Kim, Y. J.; Yoon, Y. J., Electron. Lett., 2007, 43,7 . 